METASTASIS

\title{
Elf represses snail
}

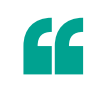

the suppression

of EMT by

ELF5 through

the repression

of SNAI2

expression

can reduce

breast cancer

metastasis to

the lung
The process of epithelial to mesenchymal transition (EMT) is thought to be essential for the invasive behaviour of epithelial cancers. Satrajit Sinha, Yibin Kang and colleagues show that the ETS transcription factor family member ELF5 (also known as ESE2) can suppress EMT in breast cancer cells through the repression of SNAI2 (also known as SLUG), a known inducer of EMT.

Expression of ELF5 is required for normal alveolar morphogenesis and lactation during pregnancy in mice, and its expression is often lost in human breast cancers and in human breast cancer cell lines. Kang and colleagues examined mammary epithelial cells during pregnancy in Elf5-knockout mice and found that the mammary epithelial cells had reduced E-cadherin staining and increased expression of SNAIL2 compared with wild-type mammary epithelial cells. Gene expression analyses of Elf5-deficient mammary epithelial cells indicated the increased expression of genes known to be involved in promoting EMT, such as Twist 1, Twist2, Zeb1, Zeb2 and Snai2. Moreover, gene-set enrichment analyses indicated that four distinct EMT-cancer stem cell gene signatures were increased in Elf5-knockout mammary epithelial cells.

To biologically verify these findings, the authors overexpressed ELF5 in mouse mammary epithelial cells and showed that these cells were blocked from undergoing EMT in vitro in response to treatment with transforming growth factor- $\beta$ (TGF $\beta$ ), and that increased expression of genes associated with the mesenchymal phenotype, including the TWIST and ZEB genes, was not evident in cells overexpressing ELF5. They also examined ELF5 expression in a panel of breast cancer cell lines and found that the cell lines that had a more mesenchymal morphology and high metastatic potential had reduced expression of ELF5. Knockdown of ELF5 using small interfering RNAs (siRNAs) in a breast cancer cell line with epithelial morphology resulted in EMT-like changes and increased motility in response to prolactin. Conversely, overexpression of ELF5

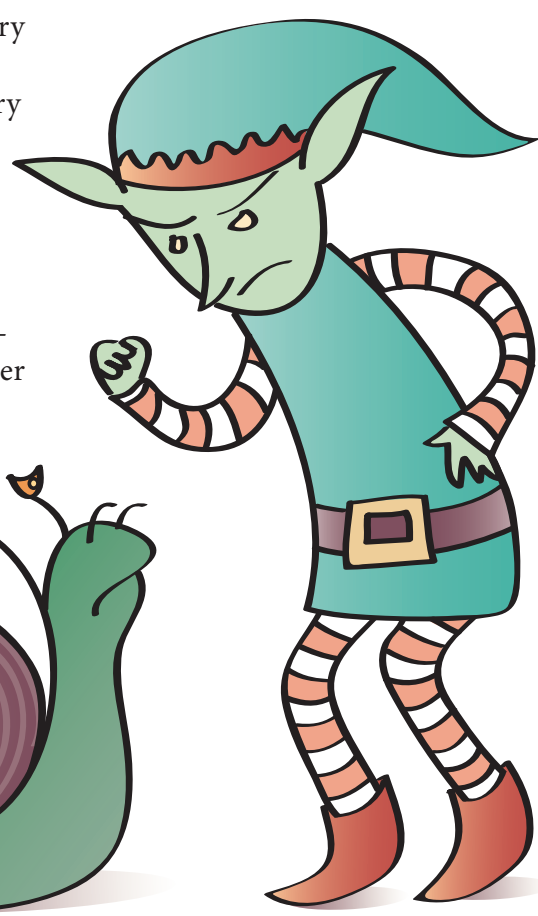

in MDA-MB-231 breast cancer cells that have a mesenchymal-like morphology induced mesenchymal to epithelial transition (MET), reduced cell motility in response to serum and substantially reduced the expression of SNAIL2. ELF5 siRNA reversed these changes, indicating that they are ELF5 driven.

Is SNAI2 a direct target of ELF5? Chromatin immunoprecipitation and quantitative PCR indicated that ELF5 binds to the proximal promoter of SNAI2. ELF5 was not detected at the promoter regions of genes such as SNAI1 or TWIST1, indicating that the effects of ELF5 are mediated by its repression of SNAI2. Indeed, overexpression of SNAIL2 in ELF5-overexpressing MDA-MB-231 cells reinstated the mesenchymal phenotype.

The authors then investigated the effect of ELF5 on breast cancer metastasis to the lung using a variety of approaches in both nude and immunocompetent mice. Lung metastases were significantly reduced in all the models when ELF5 was overexpressed, and this effect was reversed by the overexpression of both ELF5 and SNAIL2. Therefore, the suppression of EMT by ELF5 through the repression of SNAI2 expression can reduce breast cancer metastasis to the lung. Given that the authors also observed an inverse correlation between ELF5 expression and SNAIL2 expression in human breast cancer samples, particularly in oestrogen receptor-negative tumours, these findings indicate that the ELF5-SNAIL2 axis might be a potential target for the prevention of metastasis in breast cancer.

Nicola McCarthy

ORIGINAL RESEARCH PAPER Chakrabarti, R. et al. Elf5 inhibits the epithelial-mesenchymal transition in mammary gland development and breast cancer metastasis by transcriptionally repressing Snail2. Nature Cell Biol. 21 Oct 2012 (doi:10.1038/ncb2607) 